\title{
Article \\ Joint Channel Estimation and Synchronization Techniques for Time-Interleaved Block-Windowed Burst OFDM
}

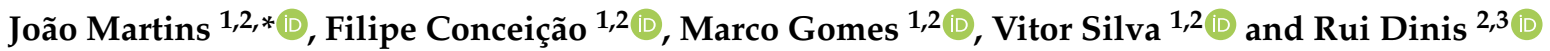 \\ 1 Department of Electrical and Computer Engineering, University of Coimbra, 3030-290 Coimbra, Portugal; \\ filipe.conceicao@co.it.pt (F.C.); marco@co.it.pt (M.G.); vitor@co.it.pt (V.S.) \\ 2 Instituto de Telecomunicações, 3810-193 Aveiro, Portugal; rdinis@fct.unl.pt \\ 3 Faculdade de Ciências e Tecnologia (FCT), Universidade Nova de Lisboa (UNL), 2829-516 Caparica, Portugal \\ * Correspondence: jm.martins@co.it.pt; Tel.: +351-967465617
}

Citation: Martins, J.; Conceição, F.; Gomes, M.; Silva, V.; Dinis, R. Joint

Channel Estimation and

Synchronization Techniques for Time-Interleaved Block-Windowed Burst OFDM. Appl. Sci. 2021, 11, 4403. https: / / doi.org/10.3390/ app11104403

Academic Editor: Alex Fragoso

Received: 22 March 2021

Accepted: 6 May 2021

Published: 12 May 2021

Publisher's Note: MDPI stays neutral with regard to jurisdictional claims in published maps and institutional affiliations.

Copyright: (C) 2021 by the authors. Licensee MDPI, Basel, Switzerland. This article is an open access article distributed under the terms and conditions of the Creative Commons Attribution (CC BY) license (https:/ / creativecommons.org/licenses/by/ $4.0 /)$.

\begin{abstract}
From a conceptual perspective, beyond-5G technologies promise to deliver very low latency, even higher data rates, and ultrareliable connections for future generations of communication systems. Modulation schemes based on orthogonal frequency-domain multiplexing (OFDM) can accommodate these requirements for wireless systems. Several hybrid OFDM-based systems, such as the time-interleaved block-windowed burst-OFDM (TIBWB-OFDM), are capable of achieving even better spectral confinement and power efficiency. This paper addresses the implementation of the TIBWB-OFDM system in more realistic and practical wireless link scenarios by addressing the challenges of proper and reliable channel estimation and frame synchronization. We propose to incorporate a preamble formed by optimal correlation training sequences such as the Zadoff-Chu (ZC) sequences. The added ZC preamble sequence is used to jointly estimate the frame beginning through signal-correlation strategies and a threshold decision device, and acquire channel-state information (CSI) by employing estimators on the basis of the preamble sequence and transmitted data. The employed receiver estimators show that it is possible to detect the TIBWB-OFDM frame beginning and highlight the robustness of the TIBWB-OFDM technique to imperfect channel estimations by showing that it can provide comparatively close BER performance to the one where the CSI is perfectly known.
\end{abstract}

Keywords: windowed OFDM; time interleaving; frame detection; frequency-domain equalization; channel estimation

\section{Introduction}

In the communications industry, wireless mobile communications are one of the most important innovations of our time due to its impact on the global economy and society. Expectations grow with the arrival and demands that the fifth generation of mobile telecommunications (5G) brings associated with reliable and quality for everywhere user access [1,2]. In [3], the 3rd Generation Partnership Project (3GPP) cemented OFDM as the preferred signal waveform in 5G, as in the previous generation, for both uplink and downlink. This is due to well-known advantages of OFDM, such as low-complexity signal processing based discrete Fourier transform (DFT) approaches, and one-tap single-carrier equalization due to the employment of cyclic prefix (CP) on transmission. Furthermore, the orthogonality between subcarriers allows for robust transmission upon severe multipath propagation. However, several studies provided alternative waveforms that are capable of outperforming some key OFDM performance indicators, the most notable being low spectral efficiency due to the use of $\mathrm{CP}$, high out-of-band radiation, and large peak-to-average power ratio (PAPR) [4,5]. Among the most recently proposed hybrid modulation waveforms, one promising candidate for beyond-5G wireless communications systems is the time-interleaved block-windowed burst orthogonal frequency division multiplexing (TIBWB-OFDM) [5-8]. Based on the BWB-OFDM [9] modulation scheme, this 
technique guarantees better spectral efficiency and low power efficiency in comparison with conventional OFDM systems. The great advantage of this approach is that it creates a diversity effect in the frequency-domain, making it possible to recover part of the lost information caused by frequency-selective channel deep fades [6]. Additionally, it can be easily implemented for multiple-input multiple-output (MIMO) and massive MIMO systems [10]. The authors in [6] proposed for the first time the TIBWB-OFDM technique, which showed considerable gains in BER performance between this new scheme and the conventional OFDM modulation. In [7], the use of nonlinear equalization was proposed at the TIBWB-OFDM receiver, namely, of the iterative block decision feedback equalization (IB-DFE) type, with considerable gains in performance. In [8], a modified version of the TIBWB-OFDM signal was proposed that included an additional overlapping operation between adjacent subsymbols, allowing for achieving even better spectral efficiency.

In this paper, we focus on the deployment of this modulation system in a more realistic scenario by addressing channel estimation and symbol synchronization problems, considering the case of severe frequency-selective channels (i.e., highly time-dispersive channels). These are, in fact, two important challenges to deal in the practical deployment of any wireless system due to channel variability along time that corrupts the transmitted signal and limits system performance [11]. Nevertheless, if the receiver is able to first correctly detect the frame beginning and accurately estimate the channel characteristics, then it is possible to recover information reliable. At the same time, we also assessed the performance of the TIBWB-OFDM technique under imperfect channel-state information (CSI), which, to the best of our knowledge, has not been studied so far.

In order to perform timing synchronization, it is important to first detect the presence of data by measuring the received signal power, and then estimate the beginning of preamble sequences [12]. This can be conducted by searching for periodic structures within the signal, i.e., applying correlation-signal techniques between the received frame and known markers. Therefore, the use of cross-correlation could provide appropriate frame detection [13]. For that, it is important to carefully choose optimal preamble sequences for the system-acquisition process. In LTE communication schemes, primary synchronization signals (PSS) are composed by Zadoff-Chu (ZC) sequences [14], complex-valued mathematical sequences with good properties to use in synchronization techniques [15].

In terms of channel estimation, the most usual approach in OFDM-based systems is either inserting known pilot symbols or training sequences in the transmitted frame [16-18]. There are several possibilities to adopt:

- Block-type pilot allocation, used in the IEEE $802.11 \mathrm{a} / \mathrm{g} / \mathrm{n}$ standard, consists of the allocation of pilots into all subcarriers in the frequency-domain. Typically, they are designed for slow-fading frequency-selective channels when the OFDM symbols' duration is much smaller than the channel coherence time [19].

- Comb-type pilot allocation, which is used, for instance, in the IEEE 802.11a WLAN standard [20], corresponds to insert pilots at predefined subcarrier locations across the entire transmission time to resist fast channel time variations between OFDM symbols. However, it is crucial to guarantee that the spacing between each pilot subcarriers is much smaller than the channel coherence bandwidth is for effective and accurate estimation.

Topologies where pilots are scattered over the time and frequency-domains can also be taken into account, enabling a good tracking relationship between the frequency selectivity and time variation of the wireless channel. Since the pilot's grid insertion is not made in all subcarriers or in fixed subcarriers across all time, better overall system performance can be achieved by reducing pilot density, thereby improving spectral efficiency [16]. 
In our work, we propose using a ZC preamble sequence to jointly perform channel estimation and frame synchronization, which can offer a close performance comparatively to the achieved with perfect CSI. Hence, this paper offers a perspective of the techniques that could perform well in the TIBWB-OFDM system, including the preamble blocktype allocation strategy, the frame correlator algorithm, and channel estimators for a linear equalizer receiver structure or by adapting the iterative block decision feedback equalization (IB-DFE) [21,22] structure.

The main contributions of our work are to:

- evaluate TIBWB-OFDM technique performance under imperfect CSI, showing the robustness of the technique; and

- propose a jointly frame synchronization and channel estimation technique for TIBWBOFDM transmissions that is able to maintain good TIBWB-OFDM spectral and powerefficiency characteristics, and to acquire reliable CSI information.

The remainder of the paper is organized as follows. Section 2 presents an overview of all required relevant scientific knowledge to provide a clearer perspective of the presented work. Section 3 introduces the proposed TIBWB-OFDM's joint channel estimation and frame synchronization strategy along with a set of adapted iterative equalizers. Section 4 presents the discussion and analysis of the results of channel estimation algorithms and frame detection for the TIBWB-OFDM system. Lastly, conclusions and future work proposals are presented in Section 5.

\section{Background}

In this chapter we introduce the background knowledge of the TIBWB-OFDM modulation systems with special attention to symbol equalization, synchronization, and channel estimation techniques.

\subsection{TIBWB-OFDM Waveform}

The building process of the TIBWB-OFDM transmitted block $\mathbf{X}$ consists of packing together a set of $N_{s}$-windowed OFDM symbols by a time-domain square-root-raised cosine (SRRC) window [23] of roll-off $\beta$, followed by a time-interleaving (permutation) operation.

Let $\mathbf{S}_{i}=\left[S_{i, 0}, S_{i, 1}, \ldots, S_{i,(N-1)}\right]^{T}$ denote the $i$ th conventional $N$-carrier OFDM symbol packed within $\mathbf{X}$, with $i=0, \ldots, N_{s-1}$, and let $\mathbf{S}=\left[\mathbf{S}_{0}, \mathbf{S}_{1}, \ldots, \mathbf{S}_{N_{s-1}}\right]$. The construction of block $\mathbf{X}$ can be represented in matrix format as

$$
\mathbf{X}=\Pi^{\left(N_{s}\right)} \operatorname{vect}\left[\mathbf{A}\left(\left(\mathbf{1}_{N_{s}}^{T} \otimes \mathbf{h}_{S R R C}\right) \odot\left(\mathbf{1}_{2} \otimes \mathbf{F S}\right)\right)\right],
$$

where

- $\quad$ F is the inverse discrete Fourier transform (IDFT) matrix with size $N \times N$;

- $\mathbf{h}_{\text {SRRC }}=\left[h_{-N}, \ldots, h_{-1}, h_{0}, \ldots, h_{N-1}\right]^{T}$ is the SRRC is the SRRC of roll-off $\beta$ where

$$
\mathrm{h}_{n}=\left\{\begin{aligned}
1, & |n| & \leq \frac{N}{2}(1-\beta) \\
\cos \left(\frac{\pi}{4 \beta}\left[\frac{2 n}{N}-(1-\beta)\right]\right), & \frac{N}{2}(1-\beta) & \leq|n|<\frac{N}{2}(1+\beta) ; \\
0, & |n| & \geq \frac{N}{2}(1+\beta)
\end{aligned}\right.
$$

- $\quad \mathbf{A}=\left[\begin{array}{lll}\mathbf{0}_{N(1+\beta) \times \frac{N}{2}(1-\beta)} & \mathbf{I}_{N(1-\beta)} & \mathbf{0}_{N(1+\beta) \times \frac{N}{2}(1-\beta)}\end{array}\right]$ is a truncation matrix that removes the trailing and ending rows of zeros that result from the windowing operation;

- $\quad \mathbf{1}_{\ell}$ is an $\ell$-length column vector of $1 \mathrm{~s}, \mathbf{0}_{m \times n}$ is $(m \times n)$-size matrix of $0 \mathrm{~s}$, and $\mathbf{I}_{n}$ is an $(n \times n)$-size identity matrix;

- $\operatorname{vect}()$ is the matrix-vectorization function that reshapes a matrix into a column by reading the matrix column by column;

- $\odot, \otimes, \times$ denote, respectively, the Hadamard, Kronecker, and conventional matrix multiplications;

- $\quad \Pi^{\left(N_{s}\right)}$ is the time-interleave permutation matrix [7]. 
Lastly, previous to transmission, a zero pad (ZP) with length $N_{Z P}$ greater than the propagation delay is added at the end of each TIBWB-OFDM block in order to avoid any intersymbol interference (ISI) [8].

\subsection{Frame Synchronization and Zadoff-Chu Sequences}

Frame detection based on the use of either pilot or preamble sequences known at the receiver is a classical strategy to achieve synchronization. Typically, these markers have proprieties that facilitate frame estimation detection [12]. Briefly, the frame beginning position is estimated by producing a correlation signal between received frame $y$ and known $L_{p}$ length preamble $p_{n}$ [13] according to

$$
\mathrm{C}_{y, p_{n}}(l)=\sum_{m} y(m) p_{n}^{*}(l+m),
$$

where $p_{n}^{*}(l)$ is the conjugate of the preamble sequence.

Depending on the chosen preamble sequence and its proprieties, when $y \approx p_{n}$, at least a high peak is produced. When performing autocorrelation, highest peak $C_{\max }$ is given by

$$
\mathrm{C}_{\max }=\sum_{m}\left|p_{n}(m)\right|^{2},
$$

where total correlation length is equal to $2 L_{p}-1$.

When the frame's estimated starting point is obtained, it is important to decide if the marked position is indeed the beginning of the frame or, e.g., define a detection probability of the following events

$$
\left\{\begin{array} { c } 
{ \mathcal { H } _ { 0 } : \text { Signal absence } } \\
{ \mathcal { H } _ { 1 } : \text { Signal detected } }
\end{array} \Leftrightarrow \left\{\begin{array}{c}
\mathcal{H}_{0}: y=\eta \\
\mathcal{H}_{1}: y=x_{n}+\eta^{\prime}
\end{array}\right.\right.
$$

where, for the absence hypothesis, the received signal is composed only by noise component $\eta$ and the detected hypothesis is the superposition between noise and transmitted signal blocks $x_{n}$.

Based on the $C_{\max }$ and noise distribution, it is important to define a threshold, $\delta_{\text {decision }}$, and a decision rule considering a length- $2 L_{p}$ sliding window analysis of (3) within each analysis. interval $\mathcal{L}$

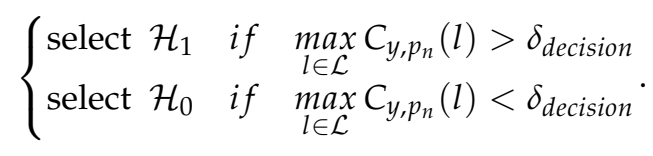

The $\delta_{\text {decision }}$ must be set in order to maximize the success frame detection probability $\left(P_{D}\right)$ and reduce situations of either false alarms $\left(P_{F}\right)$ or missed frame detection $\left(P_{M}\right)$. Mathematically, the probabilities of these definitions can be expressed as

$$
\begin{aligned}
P_{D}=P_{r}\left\{\text { Select } \mathcal{H}_{1} \mid \mathcal{H}_{1}\right\} & \times P_{r}\left\{H_{1}\right\}+P_{r}\left\{\text { Select } \mathcal{H}_{0} \mid \mathcal{H}_{0}\right\} \times P_{r}\left\{H_{0}\right\}, \\
P_{M} & =P_{r}\left\{\text { Select } \mathcal{H}_{1} \mid \mathcal{H}_{0}\right\}, \\
P_{F} & =P_{r}\left\{\text { Select } \mathcal{H}_{0} \mid \mathcal{H}_{1}\right\} .
\end{aligned}
$$

\section{Zadoff-Chu Sequences}

An essential part for achieving reliable signal recovery is the choice of optimal preamble sequences. In the LTE standard, as part of the system acquisition process, two preamble sequences defined as PSS and secondary synchronization signals (SSS) are used in the downlink process [24]. Although these SSS sequences are based on maximum-length 
sequences, also known as M sequences, the PSS is composed by Zadoff-Chu sequences [14] of odd-length period $N_{Z C}$, given by [15],

$$
z c_{n}[q]=e^{\frac{-j \pi n q(q+1)}{N_{Z C}}}, \quad q=0,1, \ldots, N_{Z C}-1,
$$

where $n$ is the root index and $n \in\left\{1, \ldots, N_{Z C}-1\right\}$.

$\mathrm{ZC}$ sequences exhibit fundamental proprieties that aid the receiver in synchronization tasks [24,25]:

- $\quad$ constant amplitude that limits the peak-to-average-power ratio (PAPR);

- "perfect" cyclic autocorrelation, i.e., correlation with its circularly shifted version is zero at different samples from $N_{Z C}$, and nonzero only at one instant, which corresponds to the $N_{Z C}$ sample. The zero-autocorrelation ZC property can be formulated by (3), resulting in

$$
\mathrm{C}_{z c, z c}[l]=\sum_{m} z c[m] z c^{*}[m+l]=P_{z c}[l],
$$

where $P_{z c}$ corresponds to the autocorrelation peak positioned in the delayed 1 sample. This is an important feature for wireless communications, enabling misaligned ZC sequences to correlate between themselves. Hence, it is possible to generate multiple orthogonal sequences just by shifting the $\mathrm{ZC}$ sequence;

- If we guarantee that the Zadoff-Chu sequence length $N_{Z C}$ is a prime number, then the cross-correlation of these two sequences is $1 / \sqrt{N_{Z C}}$.

Sequences that combine the first two properties are designated as constant amplitude zero-autocorrelation (CAZAC) sequences [25]. A crucial application of ZC sequence is for time synchronization due to their CAZAC properties. Therefore, the receiver, by analysing the sequence correlation peak inside a timing slot, can easily identify the transmitted frame beginning [26].

However, ZC sequences are affected by channel fading delay effects, resulting in additional time-domain correlation peaks. Furthermore, the correct sequence correlation peak amplitude is attenuated at high Doppler frequencies, which can lead to false detection or missing the frame beginning from the several time-delay correlation peaks, causing miscalculations of the total channel delay, consequently worsening overall system performance [15].

\subsection{Equalization and Channel Estimation}

The key receiver tasks are to perform equalization and unformat the received block. The latter operation is established by executing the opposite of the transmitter scheme, i.e., performing block time deinterleaving, matched filtering, demodulation, and channel decoding. The received TIBWB-OFDM block is converted into the frequency-domain through a DFT operation, and, assuming that the used ZP is long enough to avoid ISI, frequency-domain equalization (FDE) is executed: either linear FDE such as zero-forcing (ZF) or minimum mean square error (MMSE) methods or nonlinear iterative equalization block-based methods such as the IB-DFE [7] may be employed at this stage. When it comes to the FDE of the TIBWB-OFDM symbol, this has several advantages over time-domain equalization (TDE) in outdoor high-mobility propagation environments and for channels with severe delay spreads, since the receiver complexity can be kept low [27]. In channels of which the impulse responses remain constant within one transmitted symbol period, frequency-domain received TIBWB-OFDM signal $Y_{k}$ at each subcarrier $k=0, \ldots, N_{b}-1$ (with $N_{b}=N_{s} \times N(1+\beta)$ ) can be expressed as

$$
Y_{k}=H_{k} X_{k}+N_{k}
$$

where $H_{k}$ represents the channel frequency response (CFR). By employing linear FDE, the estimated signal is given by

$$
\hat{X}_{k}=F_{k} Y_{k}
$$


where $F_{k}$ is the frequency response of the feedforward equalization filter.

The two most popular linear equalization schemes are the ZF and MMSE equalizers. The ZF equalizer simply uses the inverse of the CFR, i.e.,

$$
F_{k}=\frac{H_{k}^{*}}{\left|H_{k}\right|^{2}}=\frac{1}{H_{k}}
$$

with the received signal given by

$$
\hat{X}_{k}=\frac{H_{k} X_{k}}{H_{k}}+\frac{N_{k}}{H_{k}}=X_{k}+\frac{N_{k}}{H_{k}} .
$$

Although its computational simplicity, this equalization technique results in noise enhancement caused on the term $\frac{N_{k}}{H_{k}}$, especially in the carriers that suffer deep fading.

An MMSE receiver tries to minimize $E\left\{\left|\hat{X}_{k}-X_{k}\right|^{2}\right\}$ by taking SNR component, $\gamma$, into account. Therefore, the equalization weight on the subcarrier $k$ is given as

$$
F_{k}=\frac{H_{k}^{*}}{\left|H_{k}\right|^{2}+\frac{1}{\gamma}}
$$

resulting in

$$
\hat{X}_{k}=\frac{H_{k}^{*} H_{k} X_{k}}{\left|H_{k}\right|^{2}+\frac{1}{\gamma}}+\frac{N_{k} H_{k}^{*}}{\left|H_{k}\right|^{2}+\frac{1}{\gamma}}=\frac{\left|H_{k}\right|^{2} X_{k}}{\left|H_{k}\right|^{2}+\frac{1}{\gamma}}+\frac{N_{k} H_{k}^{*}}{\left|H_{k}\right|^{2}+\frac{1}{\gamma}} .
$$

This equalizer has the advantage of minimizing the noise-enhancement problem in low SNR regimes, although it does not make a perfect inversion of the channel. When the SNR is high enough, it is also clear from (17) that the MMSE equalizer approaches the zero-forcing equalizer [19].

However, performance is far from that of the matched filter bound (MFB), so the alternative approach arrives by adopting nonlinear schemes such as decision feedback equalizers [28]. The IB-DFE equalizer is particularly suited for block-based single-carrier (SC) transmissions, and can deal with ISI and interblock interference (IBI) [7] by means of both a feedforward filter $\left(F_{k}^{l}\right)$ that acts as a conventional FDE in order to decrease precursors created by the wireless channel, and a feedback filter $\left(B_{k}^{l}\right)$ that attempts to cancel the remaining interferences through information from previous precursors.

Note that in the case of a TIBWB-OFDM transmission, the whole received block is first processed as being a type of a block-based SC transmission [6]. By employing IB-DFE, the equalized TIBWB-OFDM symbol is given by

$$
\tilde{X}_{k}^{(l)}=F_{k}^{(l)} Y_{k}-B_{k}^{(l)} \hat{X}_{k}^{(l-1)},
$$

and $\hat{X}_{k}^{(l-1)}$ denotes the frequency-domain signal estimated at the previous iteration by implementing either a hard or soft feedback filter decision [7]. The optimal feedforward and feedback filter coefficients are, respectively, given by

$$
F_{k}^{l}=\frac{\kappa H_{k}^{*}}{\frac{1}{\gamma}+\left(1-\left(\rho_{b l k}^{l-1}\right)^{2}\right)\left|H_{k}\right|^{2}}
$$

and

$$
B_{k}^{l}=\rho_{b l k}^{l-1}\left(F_{k}^{l} H_{k}-1\right),
$$

where $\kappa$ denotes a normalized constant chosen to guarantee that $\frac{1}{N} \sum_{k=0}^{N-1} F_{k}^{l} H_{k}=1$, and $\rho_{b l k}$ represents the correlation factor, a key parameter for reliable IB-DFE operation and accurate system performance $[7,22]$. 
A challenging obstacle in any communication system for the success of the equalization procedure is the demanded necessity for the receiver to obtain the CSI and frequently updates on it, which is not an easy task due to the time variance and frequency selectivity of wireless channels. The pilot sequence length should, therefore, be optimized considering the CIR length with the objective of reducing channel estimation errors, the BER, and maximizing channel capacity [29]. Channel estimation techniques can be classified into two categories [19,30]:

- non-data-aided, where the CSI is obtained without the use of reference training signals, i.e., on the basis of the statistics of received signal sequences; and

- data-aided, which require added information, i.e., reference training signals that are included in the transmitted frame.

Although non-data-aided techniques do not require reference signals, a large number of data must be collected in order to obtain reliable estimation channel samples. Hence, data-aided techniques, although the require additional frame overhead on the transmitted data that causes a decrease in spectral efficiency, can provide better performance, especially on fast-changing channel conditions.

The simplest method for frequency channel estimation is to use block-type allocation and employ least-square (LS) estimation $\hat{H}_{L S}$, where it is necessary for each $k^{\text {th }}$ subcarrier to compute [11]

$$
\hat{H}_{L S_{k}}=\frac{Y_{k}}{X_{k}}=H_{k}+\frac{N_{k}}{X_{k}} .
$$

The LS estimator constitutes a low-complexity algorithm. However, the major disadvantage comes from noise enhancement. Since LS estimators do not generally require any channel statistics, the estimation is not perfect enough, especially in scenarios of very fast channel variations where system performance significantly deteriorates [18]. Nevertheless, it is essential to obtain for an initial coarse channel estimation.

\section{Synchronization and Channel Estimation for TIBWB-OFDM}

In order to perform signal synchronization and channel estimation tasks for TIBWBOFDM, both transmitter and receiver schemes presented in [6] need to be improved for practical deployment. The two following subsections explain the proposed transceiver architecture in more detail.

The inherent characteristics of the TIBWB-OFDM scheme favor the adoption of a preamble allocation strategy due to the packing of several OFDM symbols into a single block. So, as shown in Figure 1, the interleaved block vector is attached to the $N_{p}$-length preamble sequence $S_{Z C}$, forming a new TIBWB-OFDM frame block.

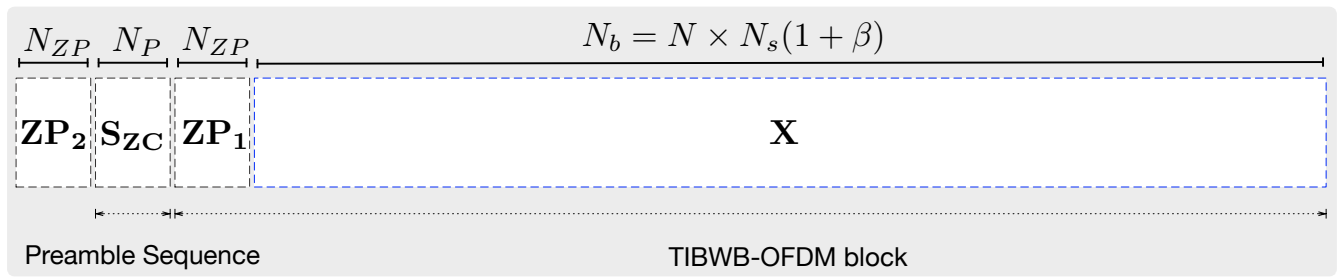

Figure 1. Adopted TIBWB-OFDM block frame structure.

Due to their autocorrelation properties and low PAPR, ZC sequences already employed in LTE are a good candidate. With an additional ZP, the same sequence can be used for CSI estimation. Since a single ZC sequence is sent per several OFDM blocks packed within the TIBWB-OFDM block, spectral efficiency suffers minimal decrease. 
Settling the transmitter block configuration, it is vital to develop the receiver architecture.

Figure 2 shows an additional new subsystem named "synchronization and channel estimators", essential to accomplish signal recovery at first instance, and frequency-domain channel estimation and equalization. It is also necessary to precisely know the transmitted preamble sequence. Thus, stored ZC preamble sequences are a key element of the receiver structure. The respective receiver scheme specifically shows equalizer implementation for the nonlinear IB-DFE type. If the desired receiver structure is a simple linear equalizer, then only TIBWB-OFDM unformatting is performed, obtaining the final estimated sequence as a one-iteration transceiver system. Afterwards, the TIBWB-OFDM structure is composed of equalizers and block unformatting, i.e., block time deinterleaving, bit deinterleaving, demapping, and channel decoding.

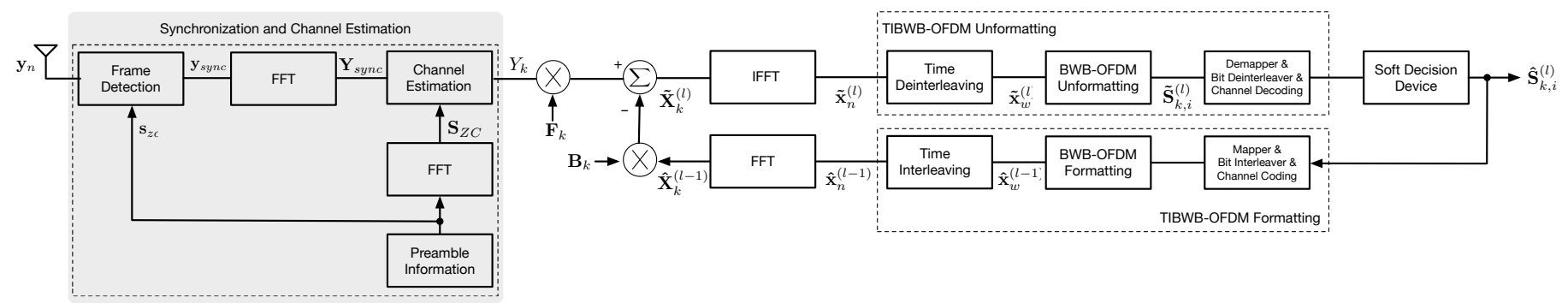

Figure 2. Preamble-assisted TIBWB-OFDM receiver scheme with IB-DFE equalization.

\subsection{Frame Synchronization}

Taking advantage of ZC proprieties, a time-domain correlation operation can be performed between the received preamble signal and known sequence in order to obtain the beginning of each transmitted frame. Hence, by autocorrelating the known preamble sequence, maximal correlation peak $P_{z c}$ is expected to appear in the $N_{Z C}$ sample. This value is extremely important since it defines the threshold percentage value for the receiver system markers, thereby affecting the success or miss probability of detection. Figure 3 shows the frame-detection algorithm flow graph.

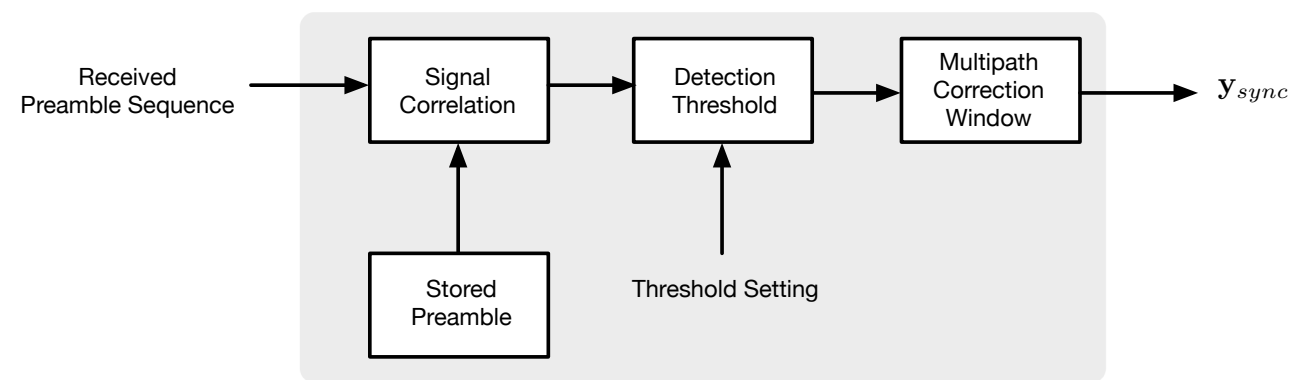

Figure 3. Frame-detection algorithm procedure for ZC preamble in TIBWB-OFDM receiver system.

In order to better understand Figure 3, Algorithm 1 details in pseudocode the followed procedure for frame detection. Input variables are the TIBWB-OFDM modulated block $\mathbf{X}$, composed by a Zadoff-Chu preamble sequence, $\mathbf{S}_{\mathbf{Z C}}$, and threshold setting parameter $\delta_{\text {decision }}$. For the output result, it is expected to obtain correlation index peaks of several transmitted preamble frames and stored ZC sequence $y_{s y n c}$ that translates the frame beginning sequence. 
The threshold decision device implementation is not straightforward due to the wireless-channel multipath effect. So, to achieve good performance, threshold variable threshold is not fixed, and fluctuates depending on the transmitted correlation preamble amplitude peak. More than one peak may appear in dispersive channels situations due to signal echos. Therefore, after the detection threshold signal $y_{\text {thold }}$, it is necessary to develop a correction algorithm based on a moving window width $\mathcal{L}$ and the marked threshold indices $y_{\text {index }}$, that accepts the maximal peak index and eliminates the remaining ones inside the respective window. The unique amplitude peak marks the presence of the frame TIBWB-OFDM beginning. However, if we encounter other high amplitude peaks where it is not expected, the algorithm acknowledges this event has a possible noise frame, rejecting the respective block.

\subsection{Channel Estimation}

After the frame synchronization procedure, the CSI is acquired. Three algorithms were developed for estimating the CSI:

- ZC preamble-based channel estimation (Algorithm A);

- data-based channel estimation (Algorithm B); and

- combined ZC and data channel estimation (Algorithm C).

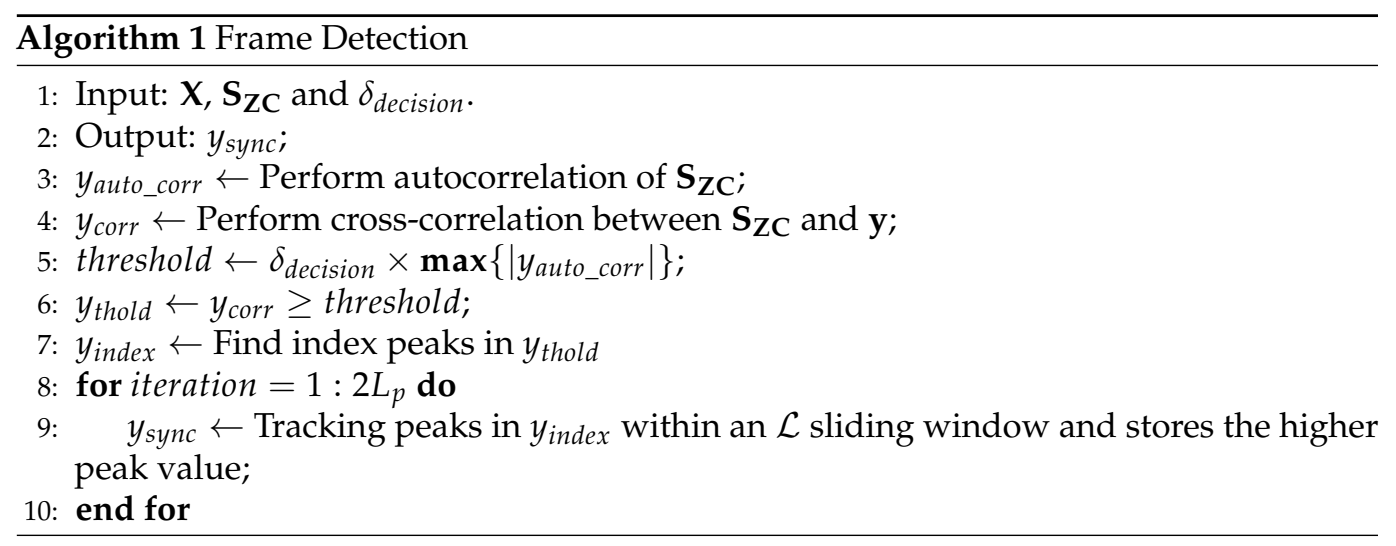

\section{Algorithm A}

Knowing the estimated $y_{s y n c}$, received samples corresponding to $[Z P, Z C]$ are used to estimate the channel. An FFT of length $N_{Z P}+N_{p}$ is applied to the received samples and divided by the corresponding transmitted samples, i.e., a LS estimator is used according to

$$
\widetilde{H}_{k(z c),\left(N_{Z P}+N_{p}\right)}=\frac{F F T_{N_{Z P}+N_{p}}\left\{\widetilde{s}_{z c}\right\}}{F F T_{N_{Z P}+N_{p}}\left\{s_{z c}\right\}}
$$

where $\widetilde{s}_{z c}$ represents the received preamble sequence. The $N_{Z P}$ sequence length is always larger than the CIR, thus enabling the reception of $\widetilde{s}_{Z C}$ without ISI. The estimated lowresolution (in frequency) CSI is then upconverted by interpolation to the length of the TIBWB-OFDM block.

$$
\widetilde{H}_{k(z c),\left(N_{b}\right)}=F F T_{\left(N_{b}\right)}\left\{I F F T_{N_{Z P}+N_{p}}\left\{\widetilde{H}_{k(z c),\left(N_{Z P}+N_{p}\right)}\right\}\right\},
$$

where $\widetilde{H}_{k(z c),\left(N_{b}\right)}$ represents the estimated channel constituted by $N_{b}$ samples. 


\section{Algorithm B}

If the IB-DFE is adopted, estimated data $\widehat{X}_{k}^{(l-1)}$ at iteration $l-1$ can be used to obtain an estimation of the channel to be used at iteration $l$. This is given by

$$
\widetilde{H}_{k(\text { data })}=\frac{Y_{k}}{\widehat{X}_{k}^{(l-1)}}
$$

where $\widehat{X}_{k}^{(l-1)}$ denotes the frequency-domain signal estimated at the previous $(l-1)$ iteration with $N_{b}$ samples.

\section{Algorithm C}

A refined strategy for achieving successively better estimations can be used by leveraging on estimations from Algorithm B. Once CSI is obtained with Algorithm A on the basis of the known preamble sequence, it makes no sense to disregard such information and consider only Algorithm B on the basis of estimated data. Instead, Algorithm C considers a weighted mean between the $\mathrm{ZC}$ channel estimated sequence (Algorithm A), and the data sequence estimated sequence (Algorithm B) can be applied as follows

$$
\widetilde{H}_{k(\text { data }+z c)}=\frac{\left(\frac{\widetilde{H}_{k(\text { data })}}{\sigma_{(\text {data })}^{2}}+\frac{\widetilde{H}_{k(z c)}}{\sigma_{(z c)}^{2}}\right)}{\left(\frac{1}{\sigma_{(\text {data })}^{2}}+\frac{1}{\sigma_{(z c)}^{2}}\right)},
$$

where $\sigma_{(z c)}^{2}$ and $\sigma_{(\text {data })}^{2}$ individually represent the mean square error between the estimated $\mathrm{ZC}$ and data sequence, respectively, and the original channel. This relation is given by

$$
\sigma_{(z c / \text { data })}^{2}=\mathbf{E}\left\{\left|H_{k}-\widetilde{H}_{k_{(z c / d a t a)}}\right|^{2}\right\}
$$

\section{Experimental Evaluation}

To test the behavior of the algorithms, two severe Rayleigh channel scenarios were considered with 8 and 32 symbol-spaced multipath channels echos (channel taps), respectively, with uncorrelated Rayleigh fading, and were used to evaluate frame-detection probability error and the channel estimator's performance through analysis of the BER of the received signal. The parameters for the TIBWB-OFDM wireless SISO model simulations are defined in Table 1.

Table 1. Simulation Parameters.

\begin{tabular}{ccc}
\hline Parameter & TIBWB-OFDM & Preamble \\
\hline Shaping pulse & SRRC & - \\
Roll-off factor & $\beta=0.5$ & - \\
Modulation & QPSK & - \\
OFDM symbols & $N_{s}=42$ & - \\
Root element & - & $n=34$ \\
Sub-block length & $N=64$ & $N_{Z C}=95$ \\
Block size & $N_{b}=4032$ & $N_{p}=96$ \\
LDPC & $(128,64)$ & - \\
Bit interleaving & 10 consecutive words & - \\
\hline
\end{tabular}

Since it was expected that successful frame synchronization would be dependent on the power of the sent $\mathrm{ZC}$ preamble, we also considered three cases where the average power of the transmitted $\mathrm{ZC}$ sequence was the same, and 3 or $6 \mathrm{~dB}$ higher than the average TIBWB-OFDM data power block. We also evaluated the impact of this feature on the channel estimators' performance. 


\subsection{Threshold Awareness Results}

The following simulations evaluate and acquire the best threshold index number that reduces miss frame probability. Therefore, 700 data frames, i.e., TIBWB-OFDM blocks, were randomly interleaved with 300 equally size blocks of additive white Gaussian noise (AWGN) with independent uncorrelated samples. All preamble signals were identical, which reflects the same transmitted high correlation peak amplitude. A critical performance variable is the selection of the threshold cross-correlation level, used to indicate the beginning of a new frame. Hence, the used value should be as accurate as possible to achieve acceptable frame detection that subsequently influences BER system performance and error awareness.

Figure 4 shows the probability of correct detection as a function of the setup threshold level. This value should be selected in the range of $25 \%-30 \%$ of the maximum of the autocorrelation $P_{z c}$, in which the probability of correct detection is almost perfect. When the threshold parameter is near $P_{z c}$, no data frames are detected because the signal suffers from fading (i.e., variable gain attenuation due to multipath effect), with the cross-correlation value hardly reaching the $P_{z c}$ value. In this case, since almost all frames are marked as noise, the probability of right detection is $30 \%$, corresponding to the fraction of sent noise frames. By settling the threshold too low, on the other hand, the opposite effect occurs, with almost all frames being detected as data. Here, coexisting noise peaks combined with those of the data sequences, both above the threshold, made it impossible to detect a single peak value, making the frame-recognition task very challenging to accomplish. However, in this scenario, a noteworthy difference was visible between the nonamplified ZC sequences and the remaining sequences. Since the threshold is defined as a percentage of $P_{z c}$, for the 3 and $6 \mathrm{~dB}$ cases, the threshold was much higher, precluding any noise frame to be marked as data; thus, the probability of correct detection was above $30 \%$.

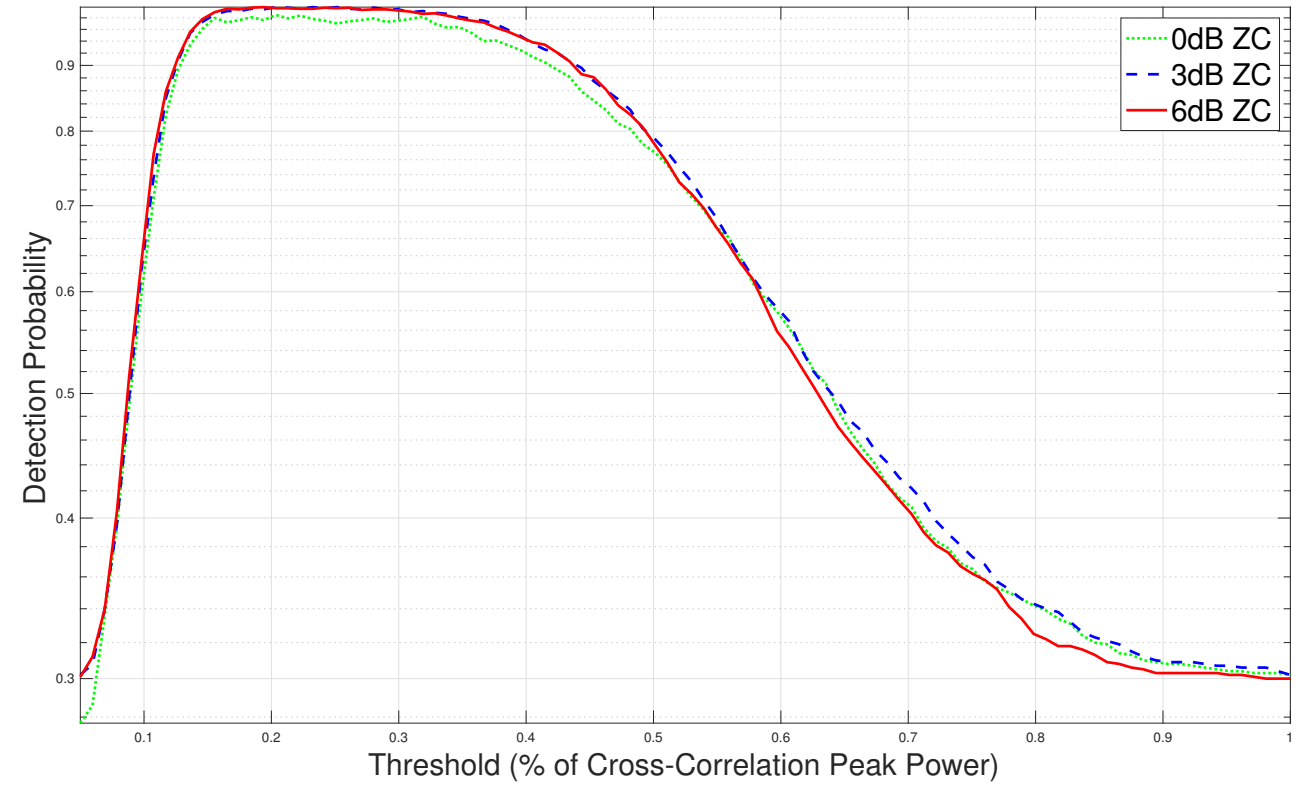

Figure 4. Correct frame detection probability as function of cross-correlation threshold value for peak detection.

\subsection{Channel Estimators with Linear Equalization}

After addressing the frame synchronization problem, we evaluated the effectiveness of the proposed CSI estimators. Therefore, subsequent simulations evaluated the BER results of the TIBWB-OFDM modulation scheme in a set condition without perfect channel-state knowledge, and compared them with the case of perfect CSI. 
Figure 5 compares TIBWB-OFDM BER performance with perfect CSI and ZC sequences as preambles in order to perform channel estimation. For a low computational scenario, Algorithm A was chosen as the first estimation approach, where the goal was to find how ZC sequences would perform in channel estimation for TIBWB-OFDM modulation with MMSE equalization.

Although the optimal CSI was not possible, good performance approximation comes with the price of necessary increased power of the preamble sequence. Having as reference the average power of the TIBWB-OFDM data block, we tested 3 situations where the appended ZC preamble was sent with the same power as data (i.e., $0 \mathrm{~dB}$ case), or $3 \mathrm{~dB}$ and $6 \mathrm{~dB}$ higher. System performance showed evident improvement with plus $3 \mathrm{~dB}$ power amplification. For $6 \mathrm{~dB}$ amplification, slight improvement was possible that almost reached the reference performance (assuming perfect synchronization and channel estimation).

Despite additional power was wasted for good system performance, this only applies to the preamble of the sub-block, which reflected approximately $2 \%$ of additional wasted total system power in the $3 \mathrm{~dB}$ scenario, and $5 \%$ in the $6 \mathrm{~dB}$ scenario for the considered TIBWB-OFDM frame structure. From a practical point of view, due to the recognized high PAPR of OFDM symbols and the TIBWB-OFDM being composed of several OFDM symbols, adding the amplified preamble did not degrade the PAPR performance since the signal's peak power was dictated by the component OFDM blocks and the robustness of the TIBWB-OFDM technique to imperfect CSI. Hence, it was not critical to increase the preamble power since the total overall power-efficiency calculation was weighted on the data frame and not on the preamble sequence.

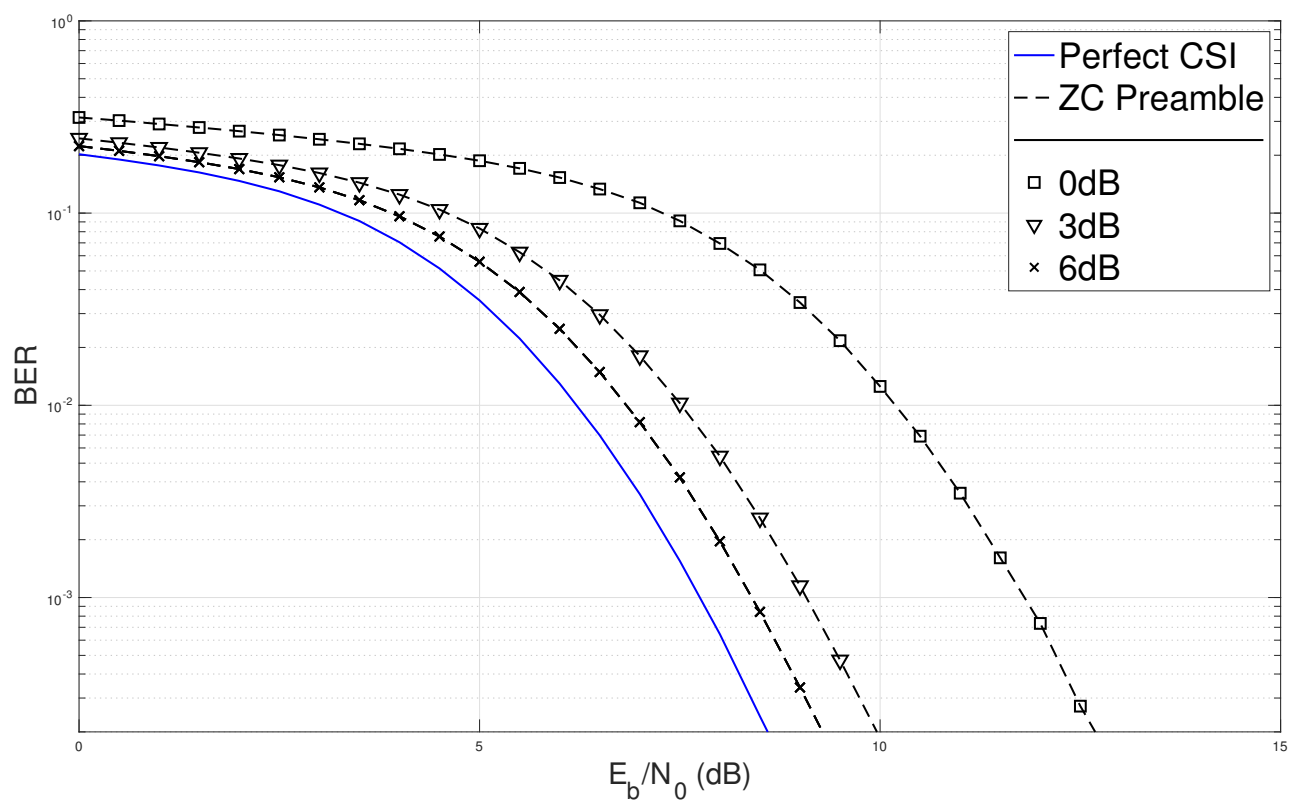

Figure 5. BER results for TIBWB-OFDM over a dispersive channel employing Algorithm A for a ZC preamble sequence with power amplification.

\subsection{Channel Estimators with Iterative Frequency-Domain Equalization}

Aiming to achieve even better estimation of the TIBWB-OFDM original bit stream, we considered the use of the IB-DFE equalizer and CSI obtained with Algorithm C, with results presented in Figures 6-8 for different values for the preamble average power. For low SNR values, the channel would maintain massive influence in mean-square-error calculation; for high SNR values, the opposite effect should happen. It should also be expected to achieve, at each IB-DFE iteration, gradually better performance. However, results showed that, even for the worst case ( $\mathrm{ZC}$ sequence with the same average power of the data is used), fine CSI estimation did not significantly affect the overall system performance. This can be justified by the good ZC performance on dispersive channels and how ineffectively the 
interference affected the preamble sequence. Also, the data did not present these good characteristics for channel estimation. So, the algorithm's estimated weight was enhanced by the first estimation performed by the ZC preamble, i.e., by Algorithm A.

Thus, although slight performance improvement was achieved, the Algorithm $C$ complexity did not justify this additional estimation procedure. However, the IB-DFE receiver showed some performance evolution from iteration to iteration, and influence in increasing the power of the preamble. Figure 8 shows the effectiveness of Algorithm A combined with IB-DFE since, with just two iterations, it could perform better than the MMSE with perfect CSI could, and a further $1 \mathrm{~dB}$ gain could be obtained if 5 iterations were considered.

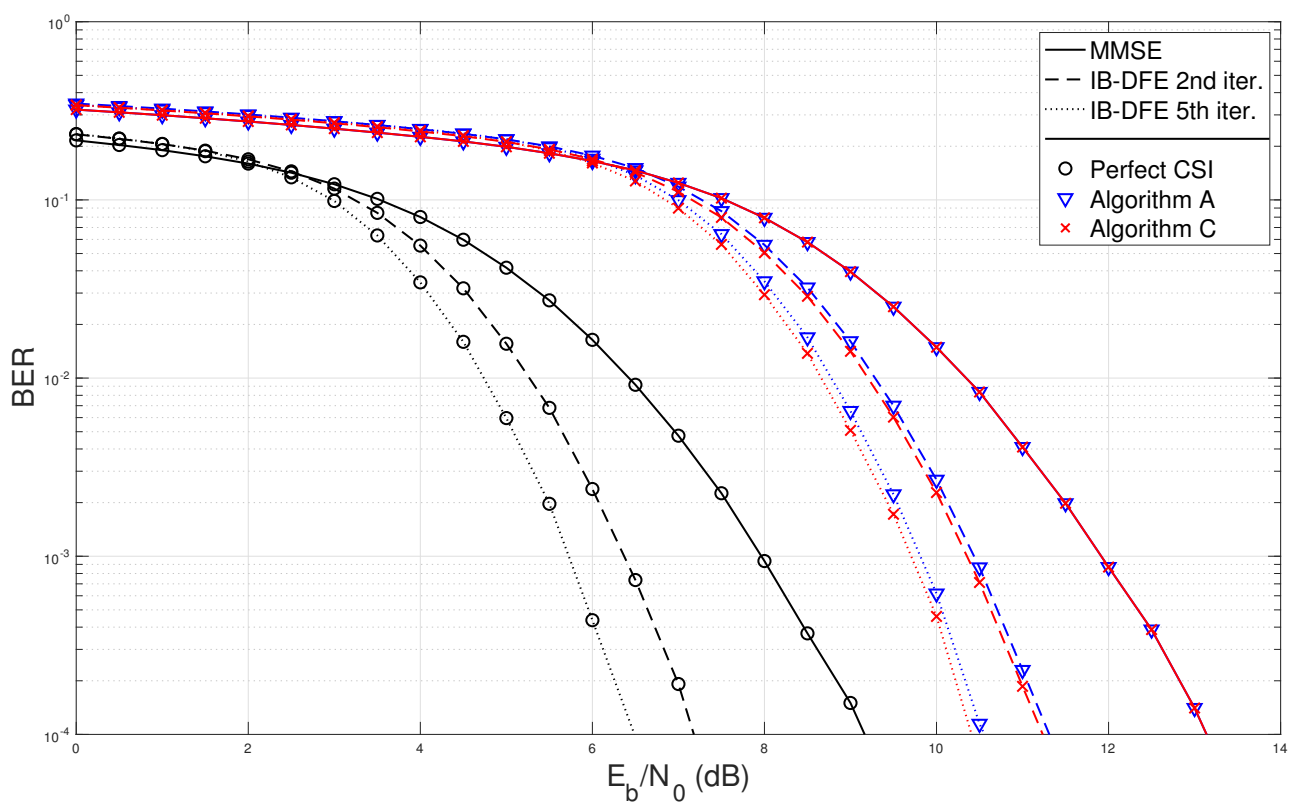

Figure 6. BER results for TIBWB-OFDM with IB-DFE over a dispersive channel employing Algorithm $\mathrm{C}$ for a $\mathrm{ZC}$ preamble having the same average power as data symbols.

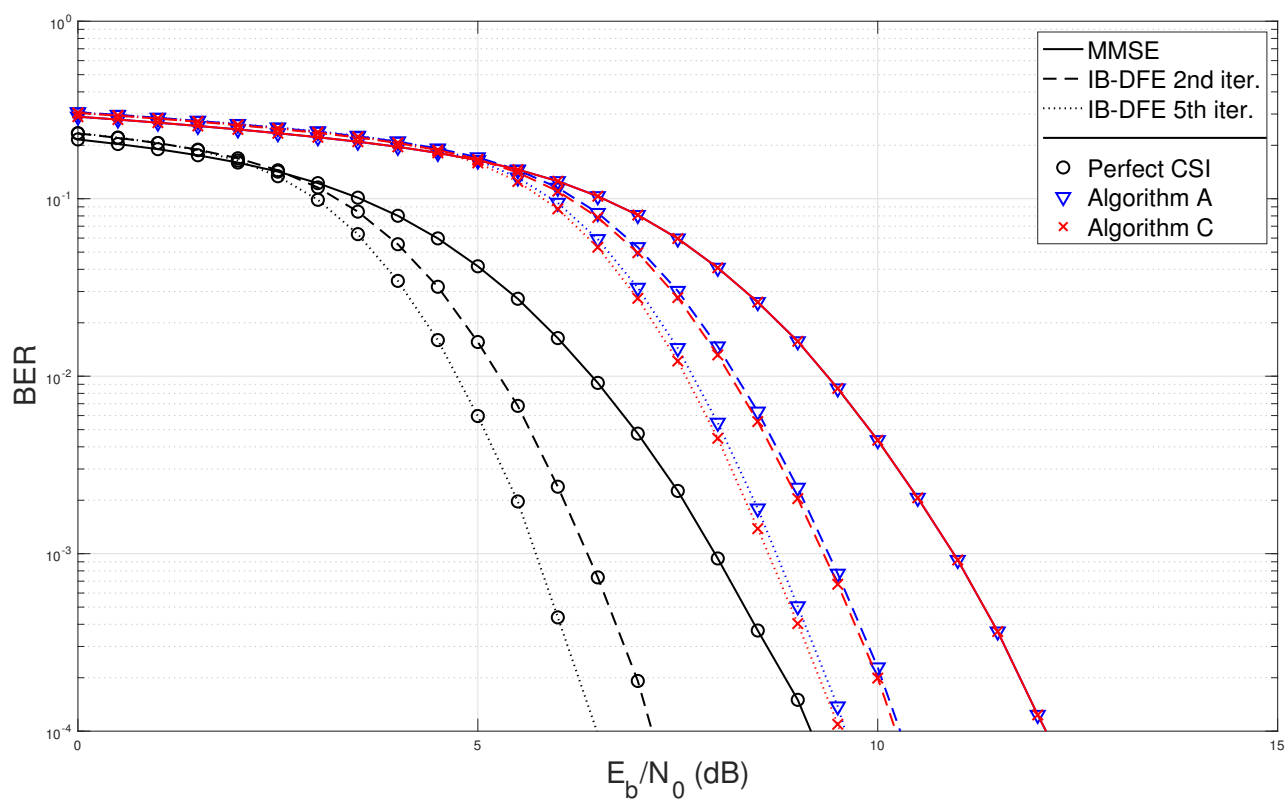

Figure 7. BER results for TIBWB-OFDM with IB-DFE over a dispersive channel employing Algorithm $\mathrm{C}$ for a $\mathrm{ZC}$ preamble sequence with $3 \mathrm{~dB}$ higher average power than the data symbols. 


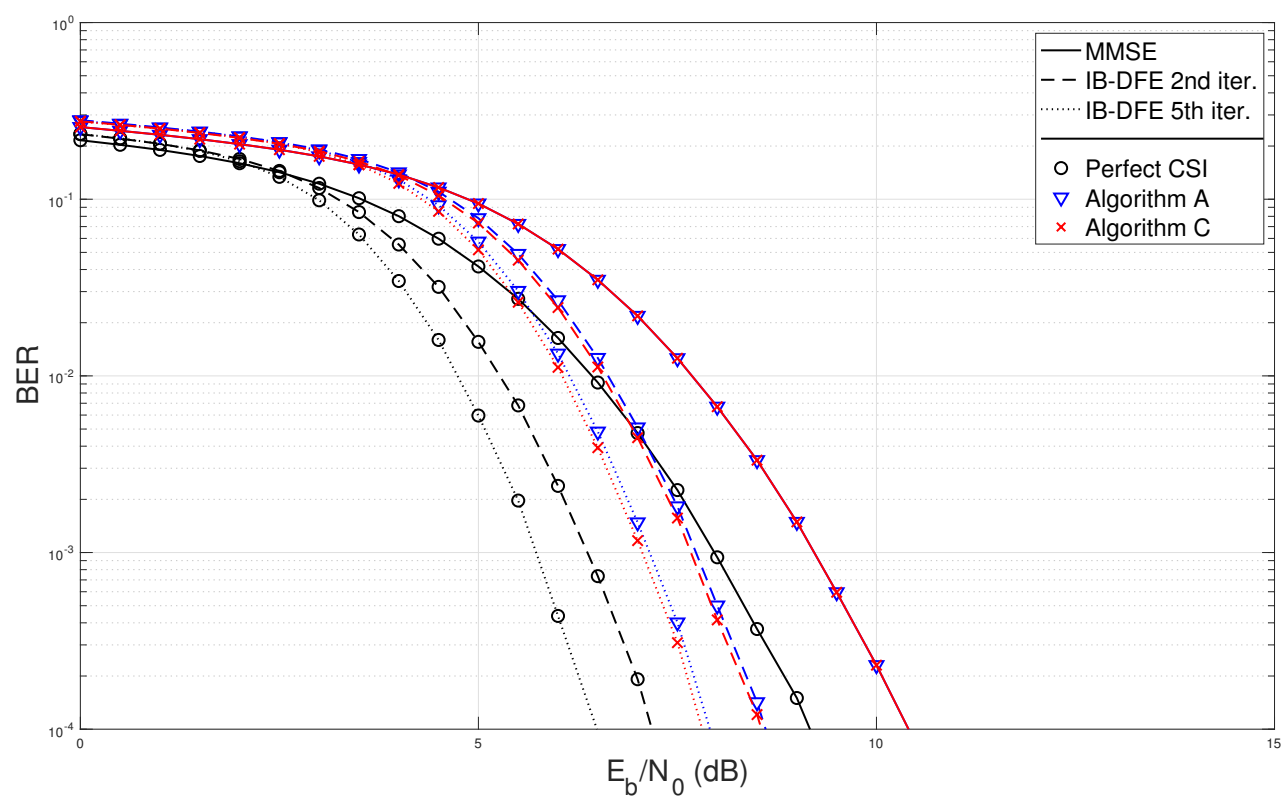

Figure 8. BER results for TIBWB-OFDM with IB-DFE over a dispersive channel employing Algorithm $\mathrm{C}$ for a $\mathrm{ZC}$ preamble sequence with $6 \mathrm{~dB}$ higher average power than the data symbols.

\section{Conclusions}

To sum up, in terms of numerical results, we could guarantee almost perfect detection by selecting a threshold in the range of $25 \%-30 \%$ from $P_{z c}$. On the other hand, employing a ZC preamble with $6 \mathrm{~dB}$ power higher than the TIBWB-OFDM data block resulted in close BER performance using both MMSE and IB-DFE equalization. Hence, the developed work constitutes a new step to provide an insight on channel estimation and frame detection algorithms that could perform well in disperse channel conditions for the TIBWB-OFDM scheme.

Our work shows that the developed estimators by means of block-type preambleassisted frames can provide close BER system performance in comparision with the true CSI performance due to the careful choice of the preamble sequences. So, it is useful to implement the pilot design based on ZC sequences due to their good correlation characteristics, and it was proven that they could jointly effectively execute channel and synchronization estimation in our hybrid modulation architecture. Nevertheless, it is necessary to guarantee that the preamble power block is amplified.

Therefore, proof of concept of how the studied modulation scheme should perform in a real-world environment was established.

Considering the obtained results, future developments should involve the study of these estimators applied to TIBWB-OFDM with windowing-time overlap [8], and the implementation of the proposed synchronization and channel estimators in a practical real environment, i.e., using a software-defined radio (SDR)-based testbed.

A future study should also compare the performance of TIBWB-OFDM with other beyond-5G waveform contenders (such as generalized frequency division multiplexing (GFDM), filter-bank multicarrier (FBMC), and filtered OFDM) under imperfect CSI.

Author Contributions: Conceptualization, funding acquisition, project administration, and supervision, M.G. and V.S.; investigation and methodology, all authors; software, J.M. and F.C.; formal analysis, J.M., M.G., and V.S.; writing—original-draft preparation, J.M.; writing-review and editing, M.G., V.S., F.C., and R.D. All authors have read and agreed to the published version of the manuscript.

Funding: This work was funded by FCT/MCTES under projects MASSIVE5G (POCI-01-0145FEDER-030588), UIDB/50008/2020-UIDP/50008/2020 and in part by the FCT Ph.D. under Grant $\mathrm{SFRH} / \mathrm{BD} / 08124 / 2020$. 
Institutional Review Board Statement: Not applicable.

Informed Consent Statement: Not applicable.

Data Availability Statement: No new data were created or analyzed in this study. Data sharing is not applicable to this article.

Conflicts of Interest: The authors declare no conflict of interest.

\begin{tabular}{|c|c|}
\hline \multicolumn{2}{|l|}{ Abbreviations } \\
\hline \multicolumn{2}{|c|}{ The following abbreviations are used in this manuscript: } \\
\hline AWGN & Additive white Gaussian noise \\
\hline BWB-OFDM & Block-windowed burst OFDM \\
\hline BER & Bit error rate \\
\hline CAZAC & Constant-amplitude zero autocorrelation \\
\hline CFR & Channel frequency response \\
\hline CIR & Channel impulse response \\
\hline CSI & Channel-state information \\
\hline $\mathrm{CP}$ & Cyclic prefix \\
\hline DFT & Discrete Fourier transform \\
\hline FDE & Frequency-domain equalization \\
\hline FFT & Fast Fourier transform \\
\hline IB-DFE & Iterative block decision feedback equalization \\
\hline IDFT & Inverse discrete Fourier transform \\
\hline $\mathrm{I} / \mathrm{Q}$ & Inphase and quadrature \\
\hline ISI & Intersymbol interference \\
\hline LTE & Long-term evolution \\
\hline MMSE & Minimum mean square error \\
\hline MIMO & Multiple-input multiple-output \\
\hline OFDM & Orthogonal frequency-division multiplexing \\
\hline PAPR & Peak-to-average Power Ratio \\
\hline PSS & Primary Synchronization Signal \\
\hline QPSK & Quadrature Phase Shift Keying \\
\hline SRRC & Square-root-raised cosine \\
\hline SNR & Signal-to-noise ratio \\
\hline SISO & Single-input single-output \\
\hline SDR & Software-defined radio \\
\hline SSS & Secondary synchronization signal \\
\hline TIBWB-OFDM & Time-interleaved block-windowed burst OFDM \\
\hline ZC & Zadoff-Chu \\
\hline $\mathrm{ZP}$ & Zero pad \\
\hline $\mathrm{ZF}$ & Zero forcing \\
\hline
\end{tabular}

\section{References}

1. Vaezi, M.; Ding, Z.; Poor, H.V. Multiple Access Techniques for 5G Wireless Networks and Beyond; Springer International Publishing: Cham, Switzerland, 2018. [CrossRef]

2. Pérez Santacruz, J.; Rommel, S.; Johannsen, U.; Jurado-Navas, A.; Tafur Monroy, I. Candidate Waveforms for ARoF in Beyond 5G. Appl. Sci. 2020, 10, 3891. [CrossRef]

3. Study on Scenarios and Requirements for Next Generation Access Technologies; Technical Report; 3rd Generation Partnership Project: France, 2017.

4. Banelli, P.; Buzzi, S.; Colavolpe, G.; Modenini, A.; Rusek, F.; Ugolini, A. Modulation Formats and Waveforms for 5G Networks: Who Will Be the Heir of OFDM?: An overview of alternative modulation schemes for improved spectral efficiency. IEEE Signal Process. Mag. 2014, 31, 80-93. [CrossRef]

5. Conceição, F.; Gomes, M.; Silva, V.; Dinis, R.; Silva, A.; Castanheira, D. A Survey of Candidate Waveforms for beyond 5G Systems. Electronics 2020, 10, 21. [CrossRef]

6. Fernandes, T.; Gomes, M.; Silva, V.; Dinis, R. Time-Interleaved Block-Windowed Burst OFDM. In Proceedings of the 2016 IEEE 84th Vehicular Technology Conference (VTC-Fall), Montreal, QC, Canada, 18-21 September 2016; pp. 1-5. [CrossRef]

7. Fernandes, T.; Pereira, A.; Gomes, M.; Silva, V.; Dinis, R. A new hybrid multicarrier transmission technique with iterative frequency domain detection. Phys. Commun. 2018, 27, 7-16. [CrossRef] 
8. Conceição, F.; Gomes, M.; Silva, V.; Dinis, R. Highly efficient TIBWB-OFDM waveform for broadband wireless communications. In Proceedings of the 2020 IEEE 91st Vehicular Technology Conference (VTC-Spring), Antwerp, Belgium, 25-28 May 2020; pp. 1-5. [CrossRef]

9. Nunes, J.; Bento, P.; Gomes, M.; Dinis, R.; Silva, V. Block-windowed burst OFDM: A high-efficiency multicarrier technique. Electron. Lett. 2014, 50, 1757-1759. [CrossRef]

10. Pereira, A.; Bento, P.; Gomes, M.; Dinis, R.; Silva, V. TIBWB-OFDM: A Promising Modulation Technique for MIMO 5G Transmissions. In Proceedings of the 2018 IEEE 88th Vehicular Technology Conference (VTC-Fall), Chicago, IL, USA, 27-30 August 2018; pp. 1-5. [CrossRef]

11. Ozdemir, M.K.; Arslan, H. Channel estimation for wireless OFDM systems. IEEE Commun. Surv. Tutor. 2007, 9, 18-48. [CrossRef]

12. Collins, T.; Getz, R.; Pu, D.; Wyglinski, A. Software-Defined Radio for Engineers; Artech House Mobile Communications Series; Artech House: Norwood, MA, USA, 2018.

13. Schmidl, T.M.; Cox, D.C. Robust frequency and timing synchronization for OFDM. IEEE Trans. Commun. 1997, 45, 1613-1621. [CrossRef]

14. Chu, D. Polyphase codes with good periodic correlation properties (Corresp.). IEEE Trans. Inf. Theory 1972, 18, 531-532. [CrossRef]

15. Dahlman, E.; Parkvall, S.; Sköld, J. 4G, LTE-Advanced Pro and The Road to 5G, 3rd ed.; Academic Press: Cambridge, MA, USA, 2016; pp. 1-590.

16. García, M.; Zazo, S.; Paez Borrallo, J. Pilot patterns for channel estimation in OFDM. Electron. Lett. 2000, 36, 1049-1050.:20000714. [CrossRef]

17. Ijiga, O.E.; Ogundile, O.O.; Familua, A.D.; Versfeld, D.J.J. Review of Channel Estimation for Candidate Waveforms of Next Generation Networks. Electronics 2019, 8, 956. [CrossRef]

18. Zhao, Z.; Cheng, X.; Wen, M.; Jiao, B.; Wang, C.X. Channel Estimation Schemes for IEEE 802.11p Standard. IEEE Intell. Transp. Syst. Mag. 2013, 5, 38-49. [CrossRef]

19. Chiueh, T.D.; Tsai, P.Y.; Lai, I.W. Baseband Receiver Design for Wireless MIMO-OFDM; Wiley-IEEE Press: Singapore, $2012 ;$ p. 360.

20. Pun, M.O.; Morelli, M.; Kuo, C.C.J. Multi-Carrier Techniques For Broadband Wireless Communications: A Signal Processing Perspective; Imperial College Press: London, UK, 2007; p. 272.

21. Benvenuto, N.; Tomasin, S. Block iterative DFE for single carrier modulation. Electron. Lett. 2002, 38, 1144-1145.:20020767. [CrossRef]

22. Benvenuto, N.; Dinis, R.; Falconer, D.; Tomasin, S. Single Carrier Modulation With Nonlinear Frequency Domain Equalization: An Idea Whose Time Has Come Again. Proc. IEEE 2010, 98, 69-96. [CrossRef]

23. Haykin, S. Communication Systems, 5th ed.; Wiley Publishing: Indianapolis, IN, USA, 2014.

24. Khan, F. LTE for 4G Mobile Broadband: Air Interface Technologies and Performance; Cambridge University Press: Cambridge, UK, 2009; doi:10.1017/CBO9780511810336. [CrossRef]

25. Sesia, S.; Toufik, I.; Baker, M. LTE-The UMTS Long Term Evolution: From Theory to Practice, 2nd ed.; John Wiley and Sons, Ltd.: Chichester, UK, 2011; doi:10.1002/9780470978504. [CrossRef]

26. Hua, M.; Wang, M.; Yang, K.W.; Zou, K.J. Analysis of the Frequency Offset Effect on Zadoff-Chu Sequence Timing Performance. IEEE Trans. Commun. 2014, 62, 4024-4039. [CrossRef]

27. Tarokh, V. New Directions in Wireless Communications Research; Springer International Publishing: Boston, MA, USA, 2009. [CrossRef]

28. Souto, N.; Dinis, R.; Correia, A.; Reis, C. Interference-Aware Iterative Block Decision Feedback Equalizer for Single-Carrier Transmission. IEEE Trans. Veh. Technol. 2015, 64, 3316-3321. [CrossRef]

29. Ohno, S.; Manasseh, E.; Nakamoto, M. Preamble and pilot symbol design for channel estimation in OFDM systems with null subcarriers. Eurasip J. Wirel. Commun. Netw. 2011, 2011, 2. [CrossRef]

30. Khaled Fazel, S.K. Multi-Carrier and Spread Spectrum Systems: From OFDM and MC-CDMA to LTE and WiMAX; Wiley: Chichester, UK, 2008; pp. 1-360. [CrossRef] 